\title{
Humans tend to walk in circles as directed by memorized visual locations at large distances
}

\author{
Patricia Consolo ${ }^{1}$, Humberto C. Holanda ${ }^{2}$, and Sérgio S. Fukusima ${ }^{1}$ \\ 1. Universidade de São Paulo, Ribeirão Preto, SP, Brazil \\ 2. Mectron - Organização Odebrecht, São José dos Campos, SP, Brazil
}

\begin{abstract}
Human veering while walking blindfolded or walking straight without any visual cues has been widely studied over the last 100 years, but the results are still controversial. The present study attempted to describe and understand the human ability to maintain the direction of a trajectory while walking without visual or audio cues with reference to a proposed mathematical model and using data collected by a global positioning system (GPS). Fifteen right-handed people of both genders, aged 18-30 years, walked without vision in an open field directly toward a target after seeing it for a brief period of time. Time and distance were directly measured by the GPS receiver. The mathematical analysis indicated that both linear and angular velocities determined the shape of the trajectory. Careful observation of the trajectories clearly showed a geometrical pattern, and the circular trajectory was the most frequent. Such behavior can be explained by the almost constant linear and angular velocity during the walk. From the perspective of the mathematical model, angular velocity is the observable physical quantity that most determines the trajectory pattern. One can notice that better performance (i.e., lower angular velocity) is related to longer distances when comparing an individual's circular trajectory walking performance as a function of different target distances (i.e., 30.00, 41.60, 57.69, and 80.00 m). Keywords: space perception, blind walking, veering, navigation.
\end{abstract}

Received 05 January 2014; received in revised form 06 May 2014; accepted 14 May 2014. Available online 25 November 2014.

\section{Introduction}

Without external cues provided by vision, maintaining a straight-ahead direction is almost impossible while walking. This tendency to deviate from an intended route while progressing under conditions of restricted environmental cues is called veering (Kallie, Schrater, \& Legge, 2007). Questions related to this natural directional bias while walking, especially its cause, have drawn the attention of researchers over the last 100 years (Guldberg, 1897; Howard \& Templeton, 1966; Mach, 1959; Schaeffer, 1928; "Why lost people walk in circles", 1893). However, such behavior is still not well understood and has raised multiple explanations. For example, the reason for this tendency toward directional walking is diversely attributable to (1) an inapt spiraling mechanism in the nervous system that is used in the absence of vision (Guldberg, 1897; Schaeffer, 1928), (2) biomechanics and

Patricia Consolo and Sérgio S. Fukusima, Universidade de São Paulo, Ribeirão Preto, Brazil. Humberto C. Holanda, Mectron, Organização Odebrecht, São José dos Campos, Brazil. Correspondence regarding this article should be directed to: Patricia Consolo, Universidade de São Paulo, Faculdade de Filosofia Ciências e Letras de Ribeirão Preto, Departamento de Psicologia, Av. Bandeirantes, 3900, Monte Alegre, Ribeirão Preto, SP, 14040-901, Brazil. Phone: +55-16-36024448. E-mail: pconsolo@pg.ffclrp.usp.br sensorial asymmetries, particularly hand and foot laterality (Day \& Goins, 1999; Scharine \& McBeath, 2002), (3) otolith system asymmetry from the fetal position (Previc \& Saucedo, 1992), (4) hemispheric asymmetry of the dopaminergic system (Mohr, Landis, Bracha, Fathi, \& Brugger, 2003; Mohr, Brugger, Bracha, Landis, \& ViaudDelmon, 2004; Mohr \& Lievesley, 2007), and (5) sensorial signal interference, in which acoustic signals, such as unpredictable noises, and postural signals can lead to greater directional deviance (Millar, 1999).

Based on these findings, there are many reasons to believe that veering cannot be adequately described by a single factor. This directional tendency ought to be a multifactor phenomenon, and such behavior emerges from an inter-relationship between central and peripheral mechanisms.

Although the literature in this field has presented divergent results and interpretations about the matter, a common aspect among all of the studies is the belief that humans, when walking blindfolded or in environments with reduced visual cues, many times involuntarily move in a large circle or spiral (Day and Goins, 1977). Indeed, this belief is present not only in the scientific literature but also in popular culture, albeit only slightly confirmed by empirical data.

Only recently have researchers sought to answer the question experimentally for longer distances. In 
analyzing the trajectories in large spaces both outdoors (Souman, Frissen, Sreenivasa, \& Ernst, 2009) and indoors (Bestaven, Guillaud, \& Cazalets, 2012), empirical evidence indicates that blindfolded subjects walk in circles, though rarely in a systematic direction.

The first goal of the present study was to identify and quantify the pattern of the observed trajectories during a walking task for which the subjects did not have visual or auditory cues over distances of $30.00,41.60,57.69$, and $80.00 \mathrm{~m}$ using a global positioning system (GPS) receiver to record the subjects' movements. We found that four different patterns could be recognized: (1) straight trajectory, (2) circular trajectory, (3) straight-circular trajectory, and (4) alternate trajectory. To understand the circular trajectory, a mathematical model was proposed to determine the geometrical quantities of this trajectory (mean radius and origin) based on physical quantities (linear speed and angular speed). This mathematical model was validated by measuring the error between the actual and predicted trajectories. Finally, we compared individual performance while walking a circular trajectory as a function of different target distances $(30.00,41.60,57.69$, and $80.00 \mathrm{~m})$.

Given that past studies on blind navigation showed that direction error varies as a function of the distance walked, we hypothesized that the increase in the target distance may lead the subject to lose directional orientation and increase the risk of expanding their angular deviations. In the particular case of the circular trajectory, an increase in angular velocities and decrease in the radius of the trajectory, indicating a worse trajectory, would be observed.

\section{Methods}

\section{Participants}

Fifteen volunteers (five male, 10 female), without any known impairment in the sensorial or musculoskeletal system, aged $18-30$ years $($ mean $=23.83$ years; $S D=$ 5.91 years), and never having previously participated in large-scale sports, participated in this study. All of the participants were right-handed and right-footed according to the Edinburgh inventory (Oldfield, 1971) and the Porac-Coren lateral preference foot questionnaire (Porac \& Coren, 1981). All of the subjects gave written informed consent prior to participation, and the study was approved by the local Ethics Committee (Process CEP-FFCLRP n ${ }^{\circ}$ 578/2011).

\section{Equipment}

The study was performed during the daytime on a level and natural grass soccer field $(90 \times 60 \mathrm{~m})$ at an educational institution between 4:00 PM and 6:00 PM to reduce sunlight cues. In this space, four egocentric distances were demarcated, defined by the physical extension between the initial position and target location. These distances $(30.00,41.60,57.69$, and $80.00 \mathrm{~m})$ were measured using a tape-measure and discreetly marked with colored golf tees.

During the experiment, the subjects were blindfolded by wearing a mask that completely occluded central and peripheral vision and equipped with headphones that generated white noise to mask acoustic cues from the environment. The movements of the participants were recorded using a Juno Handheld AS GPS (Trimble) with Terrasync Standard software (Trimble).

\section{Walking task}

Each participant was guided to the experimental space, and the researcher gave explicit instructions prior to the experiment. The participant underwent a series of practice trials, without feedback, to get used to both the equipment and the task.

At the beginning of each trial, each participant was placed at the initial point and asked to observe and memorize for a few seconds the position of the target ahead (a traffic cone, $75 \mathrm{~cm}$ height) located at a previously defined distance $(30.00,41.60,57.69$, and $80.00 \mathrm{~m}$ ). Each participant walked each distance three times, with 12 trials per subject for a total of 180 trials. The participants then wore the blindfold and headphones and walked forward at a comfortable pace and stopped when they believed they had reached the location of the target. The traffic cone was removed by an assistant and placed beside the path during the trial to avoid collision.

During the walk, the experimenter walked alongside the blindfolded participant to record the complete route with a GPS receiver and provide a safe route for the subject. The experimenter was careful to maintain the same walking pace to not influence the subject's estimates. To prevent the subjects from knowing their navigational errors and correcting their performance from one trial to the next, they were instructed to keep their blindfold on after their walk and led back to the starting point via walking a large figure 8 . Once at the starting position, the participants were instructed to briefly remove the blindfold so they could see and memorize for a few seconds (approximately $5 \mathrm{~s}$ ) the new target location, which was randomly selected, before starting the next trial.

\section{Movement registration}

The walking data (distance in meters and elapsed time in seconds) for every trial were collected and recorded using a GPS receiver, whose data acquisition frequency is $f_{\mathrm{gps}}=1 \mathrm{~Hz}$. To reduce the uncertainty of the distance, these data were extracted and post-processed using Path Finder Office software (Trimble) with differential correction and the appropriate data provided by the website of Rede Brasileira de Monitoramento Contínuo dos Sistemas GNSS, Instituto Brasileiro de Geografia e Estatística. As a result, the standard deviation of the distance measured was reduced from 2-5 $\mathrm{m}$ to $1-3 \mathrm{~m}$, values that satisfied our experimental requirements. 
After movement registration, the post-process of the data extracted from the GPS and coordinate transformation of all of the physical quantities could be calculated, namely, the subject velocity $\bar{v}$ (meters per second), angular deviation a (degrees), mean angular velocity $\dot{\psi}$ (radians per second), and radius of the theoretical circular trajectory $\boldsymbol{R}_{\mathrm{m}}$ (meters).

The subject velocity (1) was determined using the distance between two measures in each coordinate and the related time interval,

$$
\overrightarrow{\mathrm{v}}(\mathrm{t}) \approx\left(\frac{\mathrm{x}(\mathrm{t})-\mathrm{x}(\mathrm{t}-\Delta \mathrm{t})}{\Delta \mathrm{t}}, \frac{\mathrm{y}(\mathrm{t})-\mathrm{y}(\mathrm{t}-\Delta \mathrm{t})}{\Delta \mathrm{t}}\right)
$$

The velocity (in meters/seconds) is calculated at the instant t. $x(t-\Delta t)$ and $y(t-\Delta t)$ are the positions at instant $t$. and are the positions in the previous instant $\mathrm{t}-\Delta \mathrm{t} . \Delta \mathrm{t}=1 \mathrm{~s}$ is the sample time of the GPS.

To compare the subjects who had a path closer to the straight trajectory, the angular deviation was calculated according to Equation (2).

$$
\alpha=\operatorname{atan}\left(\frac{\mathrm{x}_{\mathrm{c}}}{\mathrm{y}_{\mathrm{c}}}\right)
$$

$\mathrm{x}_{\mathrm{c}}$ and $\mathrm{y}_{\mathrm{c}}$ are the local coordinates relative to the soccer field. The geometrical interpretation of this quantity is shown in Figure 1.

Lastly, we calculated both the angular velocity and mean radius of the theoretical circular path, supposing that the velocity and angular velocity of the subject are constant throughout the trajectory. In this condition, the body performs a circular trajectory. Thus, the mean radius is calculated using Equation (3).

$$
\mathrm{R}_{\mathrm{m}}=\frac{\mathrm{v}_{\mathrm{m}}}{\dot{\Psi}_{\mathrm{m}}}(3)
$$

$\mathrm{v}_{\mathrm{m}}$ is the mean velocity $(\mathrm{m} / \mathrm{s}) . \quad \dot{\psi}_{\mathrm{m}}(\mathrm{rad} / \mathrm{s})$ is the angular velocity of the body.

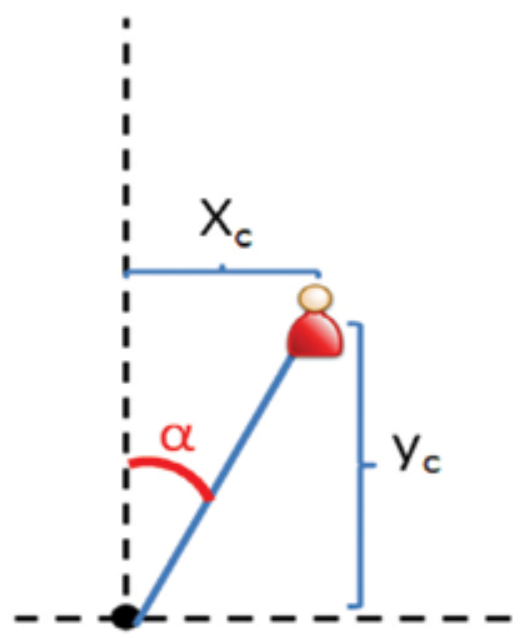

Figure 1. Geometric interpretation of an angular deviation of $\alpha$.
The circular trajectory can be described as a function of time using Equation (4).

$$
s(t)=\left(\frac{v}{\dot{\psi}}-\frac{v}{\dot{\psi}} \cos \dot{\psi} t, \frac{v}{\dot{\psi}} \sin \dot{\psi} t\right)
$$

Although the angular velocity $\dot{\psi}$ was not directly measured, its mean value $\dot{\psi}_{\mathrm{m}}$ was estimated by minimizing the functional error function $J$ between the theoretical $\left(\mathrm{x}_{\text {est }}, \mathrm{y}_{\text {ess }}\right)$ and observed trajectory given by Equation (5).

$J=\sum_{i=1}^{N}\left[x_{\text {est }}(\mathrm{i} . T)-x_{o b s}(\text { i. T })\right]^{2}+\sum_{i=1}^{N}\left[y_{\text {est }}(\text { i. } T)-y_{\text {obs }}(\mathrm{i} . T)\right]^{2}$

$\mathrm{N}$ is the total number of measured points of the trajectory. $T=1$ is the GPS sampling time.

\section{Coordinate transformation}

To describe the position of an object on the Earth's surface, one needs to use a specific coordinate system. This system is associated with the surface on which all of the calculations will be developed. The position of a body can be represented by coordinates in different ways, including on a spherical surface with denominated geodesic coordinates (such as a Geodetic System) or on a flat surface with a denominated Plane Coordinate System (such as Universal Transverse Mercator projections; Brys, 2005).

In the present study, the trajectory data were obtained by GPS and transformed into North East UP (NEU) coordinates to facilitate the analysis. Because the largest possible walking distance is less than $100 \mathrm{~m}$, the hypothesis of flat earth is satisfactory. The measurement uncertainty of each coordinate was directly obtained after post-processing, whereas the uncertainty of the calculated quantities such as velocity, total distance, and angular deviation was determined by the theory of error propagation.

Because the soccer field where the experiment was performed is not aligned with the local northeast system, the measured and post-processed coordinates were rotated through an angle of $\theta=12.73^{\circ}$ with regard to the right-hand rule applied to the vertical axis.

\section{Data calculation}

The results are presented as mean and standard deviation $( \pm \mathrm{SD})$. Nonparametric tests were also used to compare group means (Mann-Whitney test) and parameters across trials (Wilcox test). The results were considered statistically significant for $p \leq .05$.

\section{Results}

\section{Trajectory pattern}

Afterperforming the experiments for the four distances $(30.00,41.60,57.69$, and $80.00 \mathrm{~m})$ and calculating each trajectory according to the method presented in the last section above, we obtained the results for all of the 


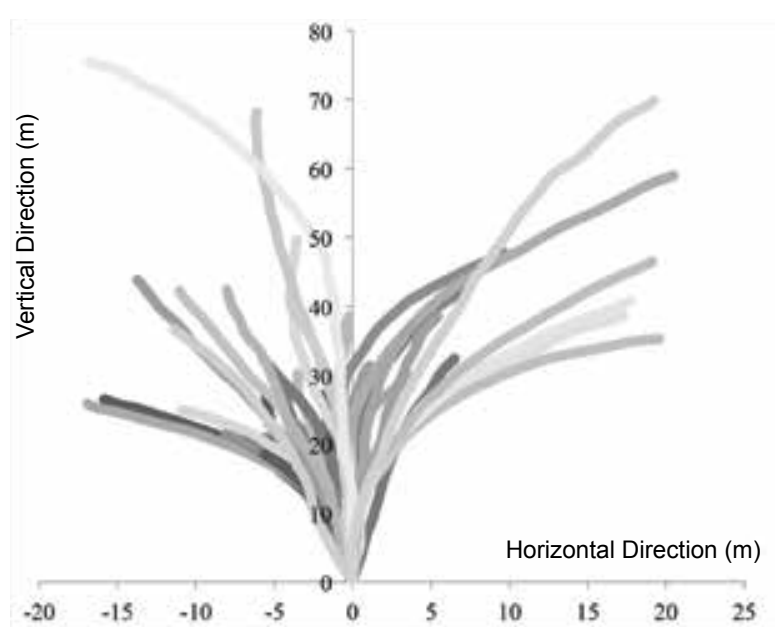

Figure 2. All trajectories during blindfolded walking to a $30.00 \mathrm{~m}$ target distance. In the frame of reference of this experiment, the horizontal direction is parallel to the field's goalposts and the vertical direction is perpendicular to them. The origin is located at the penalty spot.

subjects. Because of technical reasons, the data from one participant were excluded from the analysis. To illustrate these results, Figure 2 presents the trajectories walked relative to the target distance of $30.00 \mathrm{~m}$.

Motivated by the fact that most of the trajectories were arc of circumferences, the trajectories were classified into certain patterns. Thus, the trajectories were classified according to the following geometrical rules: (a) straight trajectory, in which the errors with respect to an ideal straight line were point-to-point less than $1.0 \mathrm{~m}$, (b) circular trajectory, in which the errors with respect to an ideal circular path were point to point less than $1.0 \mathrm{~m}$, (c) straight-circular trajectory, in which the errors with respect to an initial straight line and then a circular path were point-to-point less than $1.0 \mathrm{~m}$, and (d) alternate trajectory, in which the subject alternated between increasing and decreasing deviate trajectories. These patterns are shown in Figure 3.

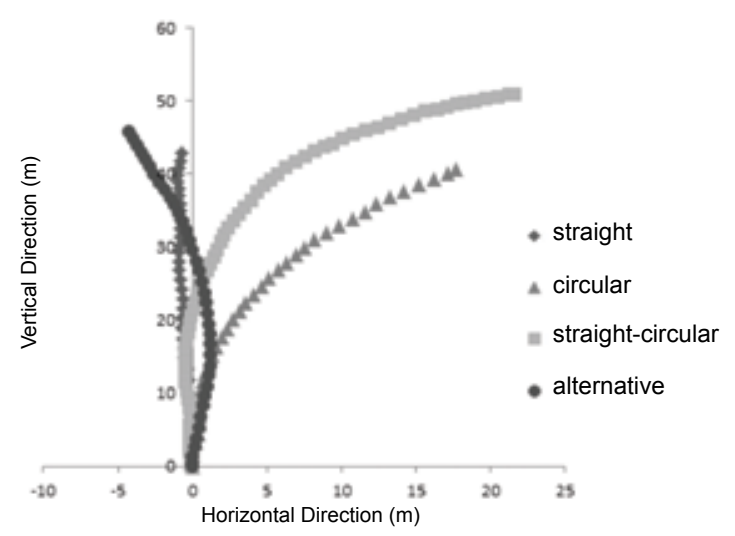

Figure 3. Example of the observed trials and their diverse classification. In the frame of reference of this experiment, the horizontal direction is parallel to the field's goalposts and the vertical direction is perpendicular to them. The origin is located at the penalty spot.
Table 1. Trajectory frequency during blind navigation (straight line, straight-circular, circular, and alternate) as a function of the target distance.

\begin{tabular}{lllll}
\hline $\begin{array}{l}\text { Target } \\
\text { distance } \\
\text { (m) }\end{array}$ & $\begin{array}{l}\text { Straight } \\
\text { line (\%) }\end{array}$ & $\begin{array}{l}\text { Straight- } \\
\text { circular } \\
(\%)\end{array}$ & $\begin{array}{l}\text { Circular } \\
(\%)\end{array}$ & $\begin{array}{l}\text { Alternate } \\
(\%)\end{array}$ \\
\hline 30.00 & 31 & 4 & 65 & 0 \\
41.60 & 7 & 14 & 71 & 7 \\
57.69 & 7 & 32 & 57 & 4 \\
80.00 & 21 & 48 & 28 & 3 \\
\hline
\end{tabular}

As shown in Table 4, for all of the distances, the most frequent pattern was the circular trajectory, and the least frequent pattern was the alternate trajectory. This assessment, based on the descriptive data, was corroborated by inferential analysis. The Mann-Whitney test for paired comparisons between the trajectory patterns indicated significant differences between the frequency of the circular pattern and the other patterns at all distances $(p<.05)$.

Table 1 also reveals some curiosities about the performance of the participants. The number of trajectories for the straight and circular patterns significantly increased with larger distances (i.e., 57.69 and $80 \mathrm{~m}$, respectively; $p<.05$ ). On average, the subjects veered later for more distant targets.

\section{Individual lateral preference}

To aid comparisons with previous and future studies that investigate veering, a score that reflects the preference in the lateral direction of deviation was established for the participants. Thus, in each trial, deviations to the right side of the body are indicated with a positive sign, and deviations to the left side of the body are indicated with a negative sign.

The majority of the participants deviated toward the left (Figure 3). The Wilcox test indicated significant lateral orientation preference to the left side $(69 \%)$ when considering the overall trials $(p<.05)$. Furthermore, more participants $(93 \%)$ changed their side of deviation between trials in one or the other direction.

\section{Circular trajectory analysis}

Visual inspection of every trial for each participant resulting from the movement of the subject while he or she was walking without being able to see the target revealed that all of the subjects deviated from the initial straight direction, performing as a general rule circular trajectories, such as circumference arcs (Figure 4).

To verify whether this directional pattern that was common among the subjects was true according to a more rigorous mathematical analysis, we attempted to fit a circle that best corresponded to the subject's trajectory. According to the explanation presented in ANNEX A, we calculated the velocity and estimated the 


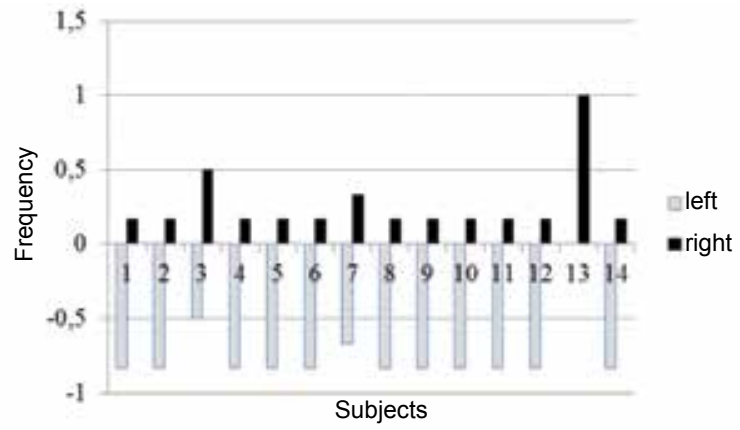

Figure 4. Veering score. Individual results are expressed in terms of the rate of deviation for all of the subjects $(n=14)$.

angular velocity and the mean radius of every trial for the four distances of the presented target. The results are summarized in Tables 1-3.

The data in Table 1 were used to verify whether the velocity and deviation varied as a function of target distance. The angular deviations were significantly greater with the large distance $(80.00 \mathrm{~m})$ compared with the shortest distance $(30.00 \mathrm{~m} ; p \leq .05)$ when considering alternate, straight, and straight-circular trajectories. No significant differences in linear velocity were found between distances $(p \geq .05)$.

When analyzing only the results of the circular trajectories shown in Table 2, we found that the average angular velocity values decreased as the target distance increased $(p \leq .05)$ and that the average radii for the target distances of 57.69 and $80.00 \mathrm{~m}$ were significantly greater than for the target distances of 30.00 and $41.60 \mathrm{~m}(p \leq .05)$. No significant differences in linear velocity or angular deviations were found between distances $(p \geq .05)$.

Interestingly, performance between trials could be assessed using the $\frac{v}{4}$ ratio. A straight trajectory has a $\frac{v}{y}$ ratio that tends toward infinity, whereas the worst trajectory has a $\frac{v}{4}$ ration that tends toward zero.

Notice that the hypotheses enumerated in the previous mathematical model are valid in this experiment. Subjects who walk on a soccer field have three degrees of freedom, given that his or her movement occurs on the plane and he or she always walks forward and not sideways. Although the hypothesis that linear speed is mainly constant during walking is not actually

Table 2. Subjects' mean (SD) velocity $\left(\mathrm{v}_{\mathrm{m}}\right)$ and angular deviation $\left(\alpha_{\mathrm{m}}\right)$ of the straight-line, straight-circular, and alternate trajectories in a blindfolded walking task as a function of the target distance.

\begin{tabular}{lllll}
\hline & \multicolumn{4}{c}{ Distance (m) } \\
\hline & 30.00 & 41.60 & 57.69 & 80.00 \\
\hline$v_{\mathrm{m}}(\mathrm{m} / \mathrm{s})$ & 1.088645 & 1.092807 & 1.080637 & 1.069234 \\
& $(.150423)$ & $(.152761)$ & $(.164306)$ & $(.130116)$ \\
\hline $\begin{array}{l}\text { Angular } \\
\text { deviation }\left(^{\circ}\right)\end{array}$ & 10.88359 & 14.43903 & 15.17768 & 16.53523 \\
& $(7.27021)$ & $(6.23411)$ & $(4.22301)$ & $(7.32321)$ \\
\hline
\end{tabular}

Table 3. Subjects' mean ( \pm SD) velocity $\left(\mathrm{v}_{\mathrm{m}}\right)$, angular deviation $\left(\alpha_{\mathrm{m}}\right)$, angular velocity $\left(\dot{\psi}_{\mathrm{m}}\right)$, and radius $\left(\mathrm{R}_{\mathrm{m}}\right)$ for circular trajectories as a function of the target distance.

\begin{tabular}{lllll}
\hline \multicolumn{5}{c}{ Distance (m) } \\
\hline & 30.00 & 41.60 & 57.69 & 80.00 \\
\hline$v_{m}(\mathrm{~m} / \mathrm{s})$ & 1.130971 & 1.117794 & 1.104549 & 1.034852 \\
& $(.140463)$ & $(.173785)$ & $(.175206)$ & $(.120995)$ \\
\hline$\alpha_{m}\left({ }^{\circ}\right)$ & 16.05234 & 16.39701 & 14.64203 & 25.36245 \\
& $(8.47061)$ & $(8.234028)$ & $(6.7311)$ & $(9.6339)$ \\
\hline$\Psi_{m}(\mathrm{rad} / \mathrm{s})$ & .022411 & .016922 & .015928 & .015392 \\
& $(.008613)$ & $(.006498)$ & $(.005049)$ & $(.005021)$ \\
\hline$R_{m}(\mathrm{~m})$ & 59.19046 & 71.44876 & 80.67353 & 84.57265 \\
& $(28.80093)$ & $(28.27592)$ & $(33.25417)$ & $(25.71603)$ \\
\hline
\end{tabular}

Table 4. Mean error $\mu$ between the coordinates of the point of the theoretical circular trajectory and corresponding point of the actual trajectory and standard deviation $\sigma$ as a function of the target distance.

\begin{tabular}{ccc}
\hline Target distance $(\mathbf{m})$ & $\boldsymbol{\mu}(\mathbf{m})$ & $\boldsymbol{\sigma}(\mathbf{m})$ \\
\hline 30.00 & .518 & .259 \\
41.60 & .587 & .348 \\
57.69 & .517 & .232 \\
80.00 & .595 & .299 \\
\hline
\end{tabular}

correct, the mean error between the theoretical and actual trajectories calculated by Equation (4) was $\mu=.554 \mathrm{~m}$, with a standard deviation of $\sigma=.285 \mathrm{~m}$ (see Table 3). The GPS uncertainty after post-processing was $1-3 \mathrm{~m}$, and the circular equation is indeed a good model to fit these data. Importantly, the greatest deviances between the theoretical and actual trajectories occurred at the end of the walk when the subjects reduced their velocity when they realized that the trajectory approached the target location. An example of such behavior can be seen in Figure 5. Even with these limitations, one can

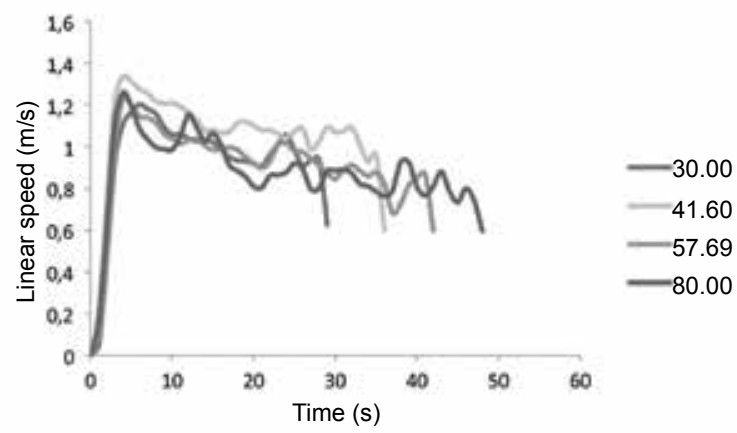

Figure 5. Examples of angular velocity plots and their observed trajectory: (a) circular, (b) straight line, (c) straightcircular, and (d) alternate. 
apply the mathematical model and eventually ascertain that some of the trajectories are approximately circular.

\section{Discussion}

\section{Angular velocity and trajectory patterns}

Although angular velocity was not directly measured, one can apply the mathematical model presented in ANNEX A along with the results of all of the experiments and intuitively predict angular velocity behavior associated with each trajectory pattern. In the discussion that follows, we assume that the subjects have considerable speed control, which was observed throughout all of the trials and is represented in Figure 5 , while their angular orientation system can freely vary as a function of time. This analysis does not seek to precisely determine the angular velocity pattern associated with a specific trajectory, but rather provide for future studies some insights into the expected behavior of angular velocity as a function of time using an intuitive approach. The results are presented in Figure 6. Likewise, with linear velocity, one can argue that the angular velocity during the beginning and end of the trajectory can likely vary greatly, and this analysis does not provide any insights into the actual angular velocity behavior. However, we should consider that these stages of the trajectory are relatively brief when considering the entire movement.

In this paper we used mathematical models to account for and quantify the observed trajectory patterns during a blindfolded walking task in a large real environment. Although this model contains specific abstractions that may be a departure from actual walking behavior, it captures relevant aspects of veering behavior.

The results demonstrated that the circular trajectory was the most frequent pattern among all of the subjects and over all of the target distances. This finding corroborates the results of previous studies (Bestaven et al., 2012; Souman et al., 2009), even under different experimental conditions.

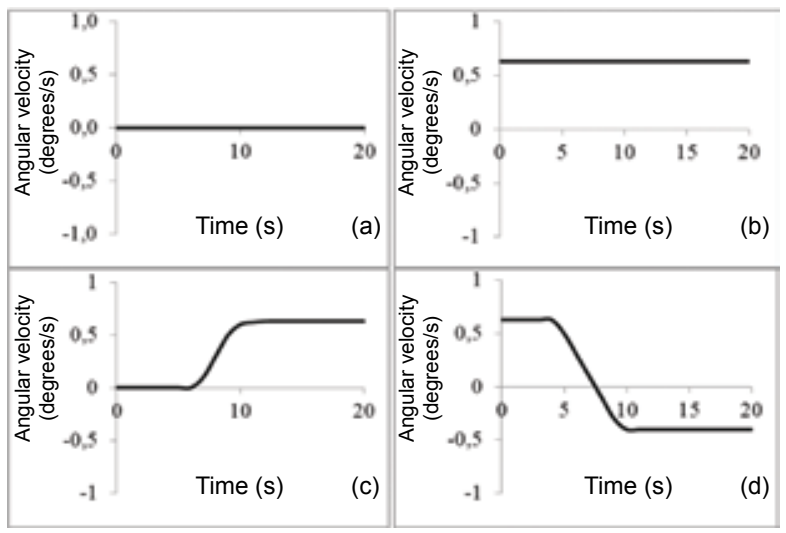

Figure 6. Speed and walking trajectories. One representative subject's performance shows linear velocity for one trial for each target distance during blind navigation.
Consequently, our results indicate that maintaining straight-ahead walking in the absence of visual and auditory information is almost impossible and that all subjects deviated toward one side or the other, unintentionally tending to walk in circles. Notice that the participants rarely corrected their trajectories during the tasks once they began. The absence of reliable sensory signals did not encourage any correction of the deviations by the subjects.

Both short and long distances require the same control of spatial orientation during navigational tasks and locomotion along the trajectory. However, if we compare individual performance when walking a circular trajectory as a function of different target distances $(30.00,41.60,57.69$, and $80.00 \mathrm{~m})$, better performance (i.e., lower angular velocity) was related to longer distances.

Given that the majority of the trajectories tended toward the left, we looked at the pattern of lateral preference as one possible reason for the individual tendency to deviate toward one side while walking. Few studies have reported that handedness or footedness is related to veering direction (Day \& Goins, 1999; Scharine \& McBeath, 2002). People who are rightfooted tend to deviate to the left side, and people who are left-footed tend to deviate to the right. Nonetheless, our results cannot support this idea when considering that our sample consisted of only right-handed individuals. Investigations of a larger group of subjects who are lefthanded and right-handed may help resolve this issue.

Because we cannot conclude that our results indicate that lateral preferences is a possible cause of this circling behavior, we should look for other possible factors, including lower to higher levels of proprioceptive and vestibular asymmetries.

A very famous theorem from geometry, Euler's rotation theorem, can provide some insights into the existence and origin of this circling behavior in the absence of vision. It states that, in a three-dimensional space, any rotation with a fixed point or a composition of rotations is identical to a singular movement around an axis. Considering that the subject walks on a flat surface, he or she can only rotate around an axis that goes approximately from his or her head to his or her feet. The rotation axis can be considered the spine, and this movement can be described by an angle. The mathematical model presented in this study considers this a viable hypothesis.

Given that any member connected to the spine can potentially have some influence on this rotational movement, slight changes in the natural head position may induce a deviation by modifying the center of locomotor activity at the spinal level (Toussaint, Do, \& Fagard, 2008).

To maintain equilibrium during locomotion, normal subjects try to stabilize their head (Pozzo, Berthoz, \& Lefort, 1990). Apparently, vision facilitates this equilibrium. Head stability most likely induces not only 
gaze stabilization but also a reference for organizing the movement of other segments (Pozzo, Levik, \& Berthoz, 1995). When vision is excluded, the vestibular system and proprioceptive information from the neck region act together to relate the trunk to space (Karnath, Sievering, \& Fetter, 1994).

Furthermore, proprioceptive input from the neck muscles and vestibular signals play an important role in building up and updating spatial representations, including the position, orientation, and movement of the body (Bottini et al., 2001). Evidence of the role of these sensory afferents comes from the specific effects of lateralized or direction-specific stimulation of peripheral sensory systems such as caloric vestibular stimulation and posterior neck muscle mechanical vibration. This stimulation can induce a distortion of egocentric coordinates, causing, for instance, unintentional deviation of the trajectory during walking (Bove, Diverio, Pozzo, \& Schieppati, 2001; Fitzpatrick, Wardman, \& Taylor, 1999) and body rotation during stepping-in-place in normal subjects (Bove, Courtine, \& Schieppati, 2002)

Therefore, the imbalance of multiple inputs that converge on the central networks that are responsible for the representation of the sagittal mid-plane would create the conditions for circling behavior (Bove et al., 2002). Although our results do not support a direct causal link between head rotation on the trunk and circling behavior, future studies should certainly elaborate on these correlations.

\section{Acknowledgements}

This work was supported by CAPES. The authors are grateful to Centro Universitário Moura Lacerda for providing the space for this study, engineer Jonas Ribeiro (Santiago e Cintra- GeoTecnologias) for help using the GPS receiver, and Maria Amélia Cesari (Universidade Federal de São João Del-Rei) for useful discussions.

\section{References}

Bestaven, E., Guillaud, E., \& Cazalets, J. R. (2012). Is "circling" behavior in humans related to postural asymmetry? PloS One, 7(9), e43861.

Bottini, G., Karnath, H. O., Vallar, G., Sterzi, R., Frith, C. D., Frackowiak, R. S., \& Paulesu, E. (2001). Cerebral representations for egocentric space: functional-anatomical evidence from caloric vestibular stimulation and neck vibration. Brain, 124(6), 11821196

Bove, M., Courtine, G., \& Schieppati, M. (2002). Neck muscle vibration and spatial orientation during stepping in place in humans. Journal of Neurophysiology, 88(5), 2232-2241.
Bove, M., Diverio, M., Pozzo, T., \& Schieppati, M. (2001). Neck muscle vibration disrupts steering of locomotion. Journal of Applied Physiology, 91(2), 581-588.

Brys, L. M. (2005). Cálculos Geodésicos - Aplicações on-line. Universidade Federal do Rio Grande do Sul, Instituto de Geociências. Retrieved from http://www.ufrgs.br/engcart/Teste/ inicial.htm; accessed May 1, 2014

Day, H. D., \& Goins, V. J. (1997). Veering in women: inconsistency of forward and backward progression. Perceptual and Motor Skills, 85(2), 587-596.

Fitzpatrick, R. C., Wardman, D. L., \& Taylor, J. L. (1999). Effects of galvanic vestibular stimulation during human walking. Journal of Physiology, 517(3), 931-939.

Guldberg, F. O. (1897) Die Cirkularbewe ung als herische Grundbewegung ihre Ursache. Phinomenahtiit und Bedeutung. eitscfri l fur Biologie, 35, 419-458.

Howard, I. P., \& Templeton, W. B. (1966). Human spatial orientation. London: Wiley.

Kallie, C. S., Schrater, P. R., \& Legge, G. E. (2007). Variability in stepping direction explains the veering behavior of blind walkers. Journal of Experimental Psychology: Human Perception and Performance, 33(1), 183-200.

Karnath, H. O., Sievering, D., \& Fetter, M. (1994). The interactive contribution of neck muscle proprioception and vestibular stimulation to subjective "straight ahead" orientation in man. Experimental Brain Research, 101(1), 140-146.

Mach, E. (1959). The analysis of sensations, and the relation of the physical to the psychical. New York: Dover.

Millar, S. (1999). Veering re-visited: noise and posture cues in walking without sight. Perception, 28, 765-780.

Mohr, C., \& Lievesley, A. (2007). Test-retest stability of an experimental measure of human turning behaviour in right-handers, mixed-handers, and left-handers. Laterality, 12(2), 172-190.

Mohr, C., Brugger, P., Bracha, H. S., Landis, T., \& Viaud-Delmon, I. (2004). Human side preferences in three different whole-body movement tasks. Behavioural Brain Research, 151(1), 321-326.

Mohr, C., Landis, T., Bracha, H. S., Fathi, M., \& Brugger, P. (2003). Human locomotion: levodopa keeps you straight. Neuroscience Letters, 339(2), 115-118.

Oldfield, R. C. (1971). The assessment and analysis of handedness: the Edinburgh inventory. Neuropsychologia, 9(1), 97-113.

Porac, C., \& Coren, S. (1981). Lateral preferences and human behavior. New York: Springer-Verlag.

Pozzo, T., Berthoz, A., \& Lefort, L. (1990). Head stabilization during various locomotor tasks in humans: I. Normal subjects. Experimental Brain Research, 82(1), 97-106.

Pozzo, T., Levik, Y., \& Berthoz, A. (1995). Head and trunk movements in the frontal plane during complex dynamic equilibrium tasks in humans. Experimental Brain Research, 106(2), 327-338.

Previc, F. H., \& Saucedo, J. C. (1992). The relationship between turning behavior and motoric dominance in humans. Perceptual and Motor Skills, 75, 935-944.

Schaeffer, A. A. (1928). Spiral movement in man. Journal of Morphology, 45(1), 293-398.

Scharine, A. A., \& McBeath, M. K. (2002). Right-handers and Americans favor turning to the right. Human Factors, 44(2), 248-256.

Souman, J. L., Frissen, I., Sreenivasa, M. N., \& Ernst, M. O. (2009). Walking straight into circles. Current Biology, 19(18), 1538-1542.

Toussaint, Y., Do, M. C., \& Fagard, J. (2008). What are the factors responsible for the deviation in stepping on the spot? Neuroscience Letters, 435(1), 60-64.

Why lost people walk in circles. (1893, February 18). Scientific American. 


\section{Appendix: Mathematical model for the circular trajectories.}

One can prove that, when moving with constant speed $v$ and constant angular speed, a body walks in a circular trajectory. Consider that a body has three degrees of freedom and can move according to two spatial coordinates and rotate over its vertical axis. In Cartesian coordinates, considering that the body starts from the origin, its velocity is expressed by (A.1).

$$
\vec{v}=(v \sin \psi t, v \cos \psi t)(\text { A. } 1)
$$

The trajectory $s(t)$ is calculated by integrating the velocity over time (A.2).

$$
s(t)=\int \vec{v} d t(A .2)
$$

Thus,

$$
s(t)=\left(\frac{v}{\dot{\psi}}-\frac{v}{\dot{\psi}} \cos \dot{\psi} t, \frac{v}{\dot{\psi}} \sin \dot{\psi} t\right)
$$

The above equation (A.3) represents a circumference of radius $R=\frac{v}{\psi}$ and center in $\left(\frac{v}{i}, 0\right)$. Indeed, this can be proven using the definition of circumference in terms of its parametric equation (A.4).

$$
\left(\mathrm{x}-\frac{\mathrm{v}}{\dot{\psi}}\right)^{2}+(\mathrm{y})^{2}=\mathrm{R}^{2} \text { (A. 4) }
$$

